\title{
Influence de la durée du forçage océano-météorologique sur la formation des seiches pour des bassins portuaires de forme rectangulaire
}

\section{Philippe SERGENT ${ }^{1}$, Jean-Michel TANGUY ${ }^{2}$, Hassan SMAOUI ${ }^{1}$}

1. Cerema, Equipe de recherche HA, 134, rue de Beauvais, CS 60039, 60280 Margnylès-Compiègne, France.

philippe.sergent@cerema.fr

2. SHF, 25, rue des favorites, 75015 Paris, France.

jm.tanguy@shf-hydro.org

\section{Résumé :}

A l'aide de l'équation intégrale de Kirchhoff - Helmholtz, nous retrouvons l'amplification ainsi que l'approximation de la période fondamentale de seiche et d'oscillation portuaire des bassins de forme rectangulaire et de profondeur constante.

En se concentrant uniquement sur l'amplification et revenant au domaine temporel, nous proposons d'abord une formulation de la réponse temporelle du résonateur à un forçage océano-météorologique quelconque. Cette réponse temporelle peut servir à estimer la réponse du bassin à un forçage instationnaire.

Nous expliquons ensuite que l'amplification maximale de la seiche est limitée par la durée $\mathrm{du}$ forçage océano-météorologique et qu'en conséquence un autre paramètre que le rapport longueur sur largeur du bassin peut intervenir contrairement aux formules classiquement proposées dans la littérature. La durée du forçage océano-météorologique limite l'amplification d'autant plus fortement que l'élancement du bassin est grand.

\section{Mots-clés :}

Kirchhoff - Helmholtz, Seiche, Résonateur, Port, Tempête, Transformée de Fourier.

\section{Introduction}

Les seiches représentent des oscillations de longues périodes de la hauteur d'un plan d'eau dans le cas de bassins semi-fermés comme les bassins portuaires. A l'aide de l'équation intégrale de Kirchhoff - Helmholtz appliquée aux ondes de gravité, nous retrouvons l'amplification et la période fondamentale de seiche pour la configuration étudiée qui est proposée notamment par RABINOVICH (2009). La formule présentée par ce dernier corrige elle-même la période classiquement utilisée pour les résonateurs quart d'onde qui est la base de la méthodologie de PONS et al. (2008). Nous rappelons les travaux de SERGENT et al. (2019) menés en mode fréquentiel. Afin de prendre en compte le forçage océano-météorologique qui est instationnaire, nous reprenons ici ces formulations en mode temporel et identifions la fonction de transfert du résonateur qui peut être utilisée pour un forçage quelconque. Nous analysons ensuite l'influence de la durée de forçage 


\section{Thème 4 - Ouvrages portuaires, offshore et de plaisance}

de la seiche sur l'amplification au fond du résonateur en fonction de l'élancement du bassin rectangulaire.

\section{Rappel des équations d'un résonateur}

Nous rappelons ici les équations de base du résonateur (SERGENT et al., 2019).

\subsection{Equation de Kirchhoff - Helmholtz}

Nous nous intéressons à un résonateur correspondant à une darse de forme rectangulaire de largeur $l$, de longueur $L$ et de profondeur constante $d$ qui est présenté sur la figure 1 dans le repère $\mathrm{Oxy}$ avec $\mathrm{O} x$ l'axe du résonateur. Les frontières sont toutes parfaitement réfléchissantes et s'étendent à l'infini sur l'axe $\mathrm{Oy}$. En appliquant l'équation intégrale de Kirchhoff - Helmholtz dans le domaine extérieur au résonateur, on peut démontrer que l'équation intégrale est l'expression de l'intégrale de Rayleigh avec $h$ la variable complexe représentant la variation du niveau d'eau par rapport au niveau moyen et $h_{i n c}$ l'excitation incidente :

$$
h(0, y)=\int_{-\frac{l}{2}}^{\frac{l}{2}} G(y \mid z) \frac{\partial h}{\partial x}(0, z) d z+h^{i n c}(0, y)
$$

La variable $z$ appartient à l'axe $\mathrm{O} y$.

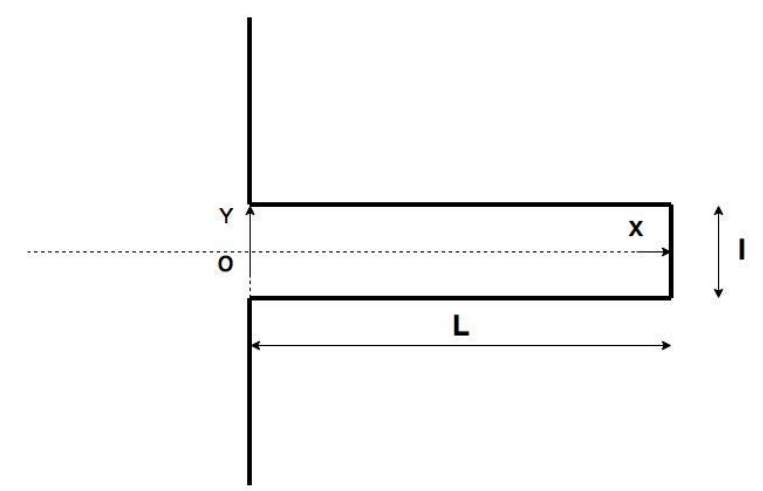

Figure 1. Géométrie du résonateur rectangulaire.

La fonction 2D de Green de l'équation de Helmholtz sur le demi-plan avec frontière réfléchissante sur Oy s'écrit :

$$
G(y \mid z)=\frac{j}{2} H_{0}^{(1)}(k|y-z|)
$$

où $H_{0}^{(1)}$ est une fonction de Hankel, $k$ le nombre d'onde et $j$ l'unité imaginaire. 


\section{XVIèmes Journées Nationales Génie Côtier - Génie Civil \\ Le Havre 2020}

L'excitation incidente s'écrit avec $a^{i n c}$ l'amplitude incidente au large :

$$
h^{i n c}(x, y)=a^{i n c}\left(e^{j k x}+e^{-j k x}\right) \sqrt{\frac{1}{l}}
$$

L'équation de dispersion donne le nombre d'onde $k$ et la célérité $c$ égale à $\frac{\omega}{k}$ avec $d$ la profondeur :

$$
\omega^{2}=g k \tanh (k d)
$$

Nous écrivons la fonction $h$ à partir du mode fondamental de propagation de l'onde :

$$
h(x, y)=\left(a_{0}^{+} e^{j k x}+a_{0}^{-} e^{-j k x}\right) \sqrt{\frac{1}{l}}
$$

En introduisant l'équation (5) dans (1) et en intégrant sur la largeur $l$ de l'entrée du résonateur, nous obtenons :

$$
a_{0}^{+}+a_{0}^{-}=j k\left(a_{0}^{+}-a_{0}^{-}\right) M_{00}+2 a^{i n c}
$$

Nous notons :

$$
M_{00}=\frac{1}{l} \int_{-\frac{l}{2}}^{\frac{l}{2}} \int_{-\frac{l}{2}}^{\frac{l}{2}} G(y \mid z) d y d z
$$

Les bornes d'intégration des variables $y$ et $z$, qui sont les ordonnées de deux points appartenant à l'entrée du résonateur, sont identiques.

\subsection{Approximation de $M_{00}$}

Dans (SERGENT et al., 2019), $M_{00}$ est approximé pour $k l \ll 1$ avec $\gamma$ le nombre d'Euler :

$$
M_{00} \approx \frac{l}{4}\left[2 j-\frac{2}{\pi}\left(2 \ln \left(\frac{k l}{2}\right)+2 \gamma-3\right)\right]
$$

\section{Estimation de la réponse temporelle d'un résonateur}

\subsection{Théorie}

Dans cet article, nous nous intéressons à la réponse temporelle du résonateur. En introduisant la fermeture au fond de la darse, alors $a_{0}^{-}=e^{2 j k L} a_{0}^{+}$.

Au fond de la darse la hauteur $h_{\text {fond }}$ s'écrit :

$$
h_{\text {fond }}=\left(a_{0}^{+} e^{j k L}+a_{0}^{-} e^{-j k L}\right) \sqrt{\frac{1}{l}}=2 a_{0}^{+} e^{j k L} \sqrt{\frac{1}{l}}
$$




\section{Thème 4 - Ouvrages portuaires, offshore et de plaisance}

L'équation (6) donne aussi :

$$
a_{0}^{+}\left[1+e^{2 j k L}-j k M_{00}\left(1-e^{2 j k L}\right)\right]=2 a^{i n c}
$$

En négligeant la partie réelle de $M_{00}$ qui ne contribue qu'à un léger déphasage temporel, l'équation (10) s'écrit finalement sous la forme :

$$
\begin{aligned}
& a_{0}^{+}\left[1+e^{2 j k L}+\frac{k l}{2}\left(1-e^{2 j k L}\right)\right]=2 a^{i n c} \\
& a_{0}^{+}=\frac{2 a^{i n c}}{1+e^{2 j k L}+\frac{k l}{2}\left(1-e^{2 j k L}\right)}
\end{aligned}
$$

Nous introduisons la fonction de transfert notée $\mathrm{G}(\omega)$ et $g(t)$ sa transformée de Fourier.

$$
\begin{aligned}
& \mathrm{G}(\omega)=\frac{h_{\text {fond }}}{h_{\text {inc }}(0,0)}=\frac{2 e^{j k L}}{1+e^{2 j k L}+\frac{k l}{2}\left(1-e^{2 j k L}\right)} \\
& \mathrm{G}(\omega)=\frac{2 e^{j k L}}{1+\frac{k l}{2}} \frac{1}{\left[1+e^{2 j k L} \frac{1-\frac{k l}{2}}{1+\frac{k l}{2}}\right]}
\end{aligned}
$$

L'équation (14) peut s'exprimer sous forme d'une série avec $k l<2$. Nous introduisons $\mathrm{F}(\omega)$ et sa transformée de Fourier $\mathrm{f}(t)$ pour simplifier.

$$
\begin{aligned}
& \mathrm{G}(\omega)=\frac{2 e^{j k L}}{1+\frac{k l}{2}} \mathrm{~F}(\omega) \\
& \mathrm{F}(\omega)=\sum_{n=0}^{\infty}(-1)^{n} e^{2 j n k L}\left(\frac{1-\frac{k l}{2}}{1+\frac{k l}{2}}\right)^{n}
\end{aligned}
$$

La fonction $\mathrm{f}(t)$ s'exprime sous la forme d'une série de termes temporels par linéarité de la transformée de Fourier :

$$
\mathrm{f}(\mathrm{t})=\sum_{n=0}^{\infty} f_{n}(\mathrm{t})
$$

Nous introduisons $\mathrm{S}(\omega)$ et sa transformée de Fourier $\mathrm{s}(t)$ :

$$
\mathrm{S}(\omega)=\frac{1-\frac{k l}{2}}{1+\frac{k l}{2}}
$$




\section{XVIèmes Journées Nationales Génie Côtier - Génie Civil \\ Le Havre 2020}

Nous avons la relation suivante avec * le symbole de la convolution et $\delta$ le symbole du

Dirac :

$$
f_{n}=-f_{n-1} * \mathrm{~s} * \delta_{\frac{2 L}{c}}
$$

L'expression (19) précise que la hauteur au fond de la darse est la superposition d'un nombre infini de réflexions à l'entrée du résonateur avec un coefficient de réflexion proche de -1 avec un amortissement caractérisé par $s(t)$. Cette expression nous permet d'estimer la réponse du résonateur avec un forçage quelconque instationnaire.

\subsection{Calcul de $s(t)$}

Pour calculer $s(t)$ nous appliquons un filtre passe bas pour la transformée de Fourier car nous nous intéressons aux ondes longues avec $k l<2$,

$$
\begin{aligned}
& \mathrm{s}(t)=\frac{1}{\pi} \int_{0}^{\frac{2 c}{l}} \frac{1-\frac{\omega l}{2 c}}{1+\frac{\omega l}{2 c}} \cos \omega t d \omega \\
& \mathrm{s}(t)=\frac{2}{\pi} \frac{c}{l} \int_{0}^{1} \frac{1-u}{1+u} \cos \left(2 \frac{c t}{l} u\right) d u
\end{aligned}
$$

Nous calculons également la transformée de Fourier $r(t)$ sans amortissement :

$$
\mathrm{r}(t)=\frac{2}{\pi} \frac{c}{l} \int_{0}^{1} \cos \left(2 \frac{c t}{l} u\right) d u=\frac{1}{\pi} \frac{\sin \left(2 \frac{c t}{l}\right)}{t}
$$

avec $\mathrm{r}(0)=\frac{2}{\pi} \frac{c}{l}$, voir figure 2 .

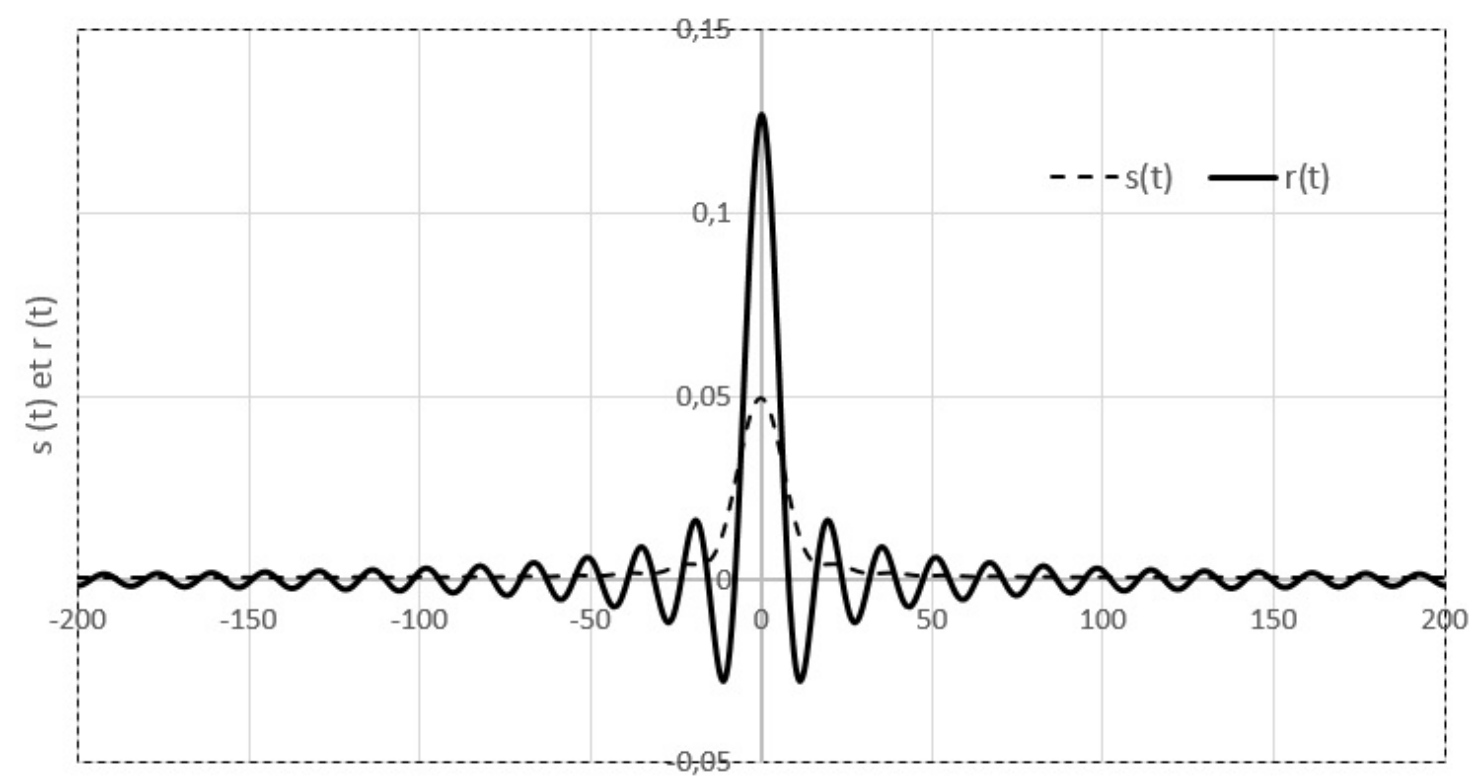

Temps $t$ en secondes

Figure 2. Transformées de Fourier pour $\frac{l}{c}=5 \mathrm{~s}$. 
Thème 4 - Ouvrages portuaires, offshore et de plaisance

\section{Application}

4.1 Validation de la réponse

Nous assimilons $g(t)$ à $2 f(t)$ en approximant l'équation (15) et introduisons $h_{\text {inc }}(t)$ l'excitation incidente.

$$
h_{\text {inc }}(t)=\sin (\omega t)
$$

En ne prenant que les deux premiers termes de l'équation (17):

$$
\begin{aligned}
& h_{\text {fond }}(t)=2 h_{\text {inc }}(t) *\left\{f_{0}(t)+f_{1}(t)\right\} \\
& h_{\text {fond }}(t)=2 \sin (\omega t) *\left\{\delta-s\left(t-\frac{2 L}{C}\right)\right\}
\end{aligned}
$$

Nous prenons $4 \frac{l}{c}=20 s ; 4 \frac{L}{c}=80 s ; \omega=\frac{\pi}{2 L} c=0,0785$, voir figure 3 .

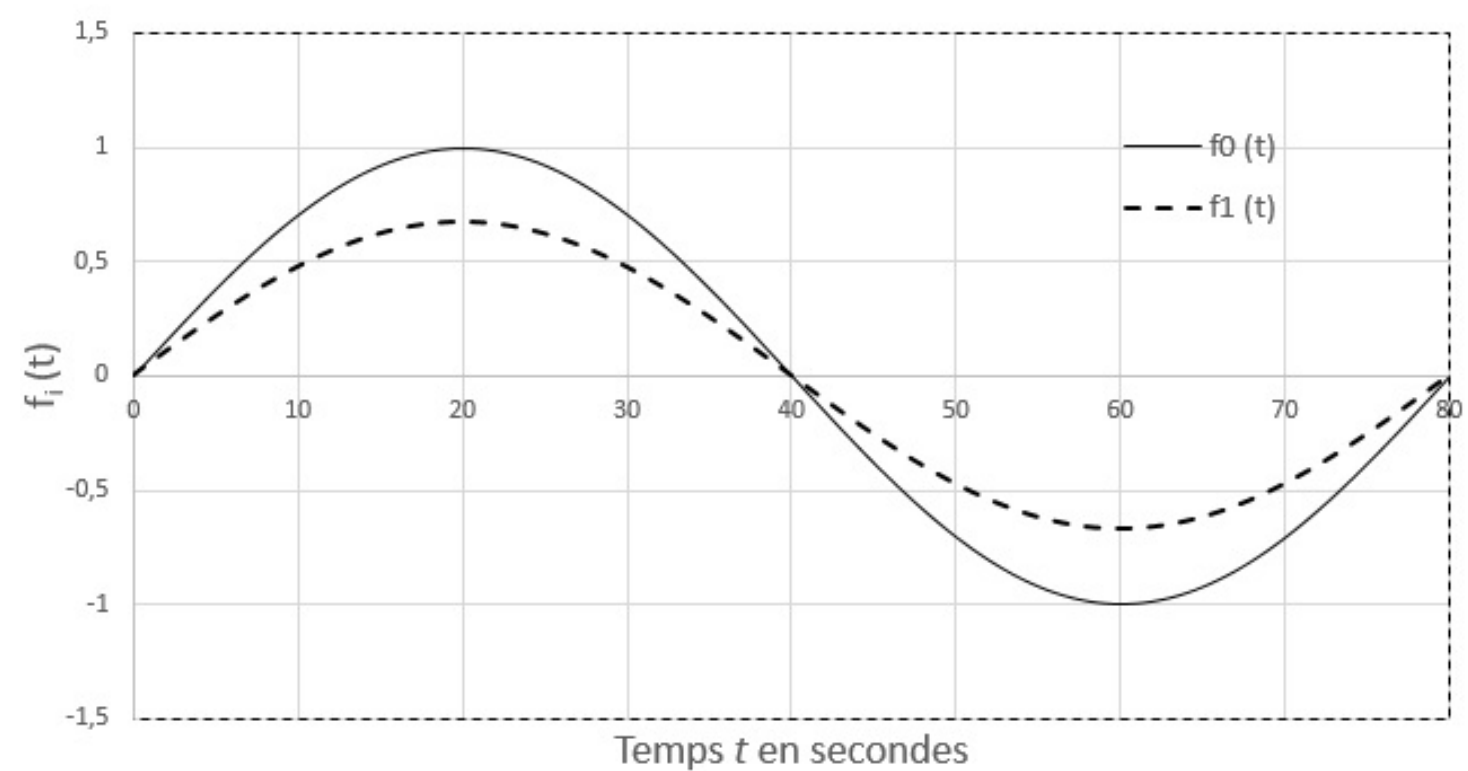

Figure 3. Fonctions $f_{0}(t)$ et $f_{1}(t)$.

Nous vérifions que l'amortissement à la première réflexion est égal à $\frac{1-\frac{k l}{2}}{1+\frac{k l}{2}}=0,672$ avec $\frac{k l}{2}=\frac{\omega}{8} \frac{4 l}{c}=0,196$.

\subsection{Généralisation}

Nous introduisons désormais la durée de la tempête $T$ égale à $n$ fois $\frac{2 L}{c}$.

$h_{\text {inc }}(t)=\sin (\omega t)$ pour $0 \leq t \leq T$

et $h_{\text {inc }}(t)=0$ en dehors de la tempête. Nous généralisons ensuite l'équation (24): 


$$
\begin{aligned}
& h_{\text {fond }}(t)=2 h_{\text {inc }}(t) * \sum_{i=0}^{n-1} f_{i}(\mathrm{t}) \\
& f_{i}(\mathrm{t}) \approx\left(-\frac{1-\frac{k l}{2}}{1+\frac{k l}{2}}\right)^{i} \delta\left(t-\frac{2 i L}{C}\right)
\end{aligned}
$$

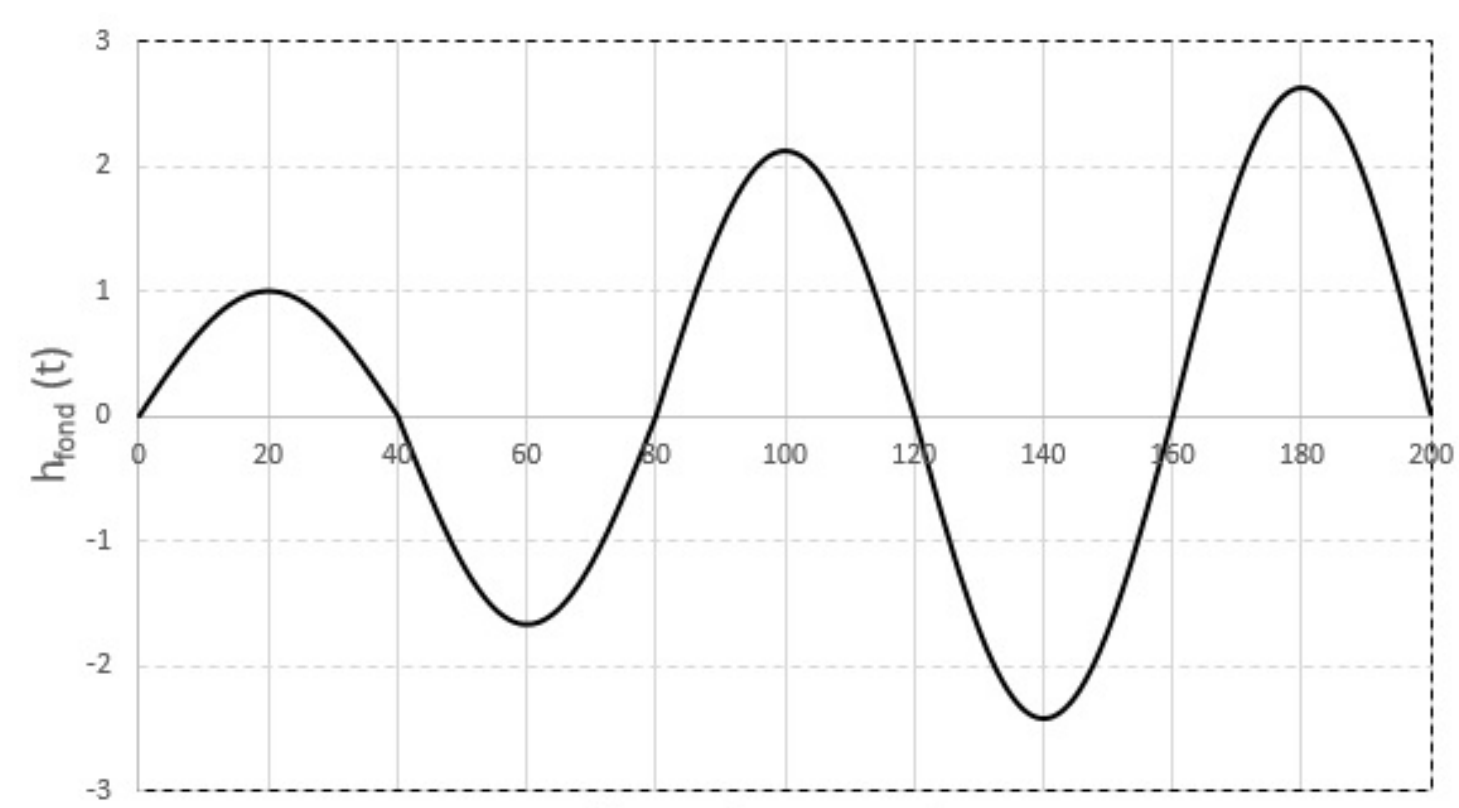

Temps $t$ en secondes

Figure 4. Evolution temporelle de $h_{\text {fond }}(t)$.

La figure 4 montre l'évolution temporelle de la hauteur au fond du bassin en fonction du temps. Nous visualisons l'augmentation de l'amplitude du signal à chaque demi-période.

4.3 Estimation de l'amplitude de la seiche en fonction de la durée de la tempête A partir de la figure 4, nous sommes en mesure d'estimer l'amplitude maximale de la seiche $A$ en fonction de la durée $T$ ou du nombre $n$.

$$
A \approx 2 \sum_{i=0}^{n-1}\left(\frac{1-\frac{k l}{2}}{1+\frac{k l}{2}}\right)^{i}=2 \frac{1-\left(\frac{1-\frac{k l}{2}}{1+\frac{k l}{2}}\right)^{n}}{1-\left(\frac{1-\frac{k l}{2}}{1+\frac{k l}{2}}\right)}
$$


Nous savons $\frac{k l \_}{2}=\frac{\pi q}{4}$ où le nombre $q$ est égal à $\frac{l}{L}$.

$$
\begin{aligned}
& A \approx \frac{4}{\pi q}\left(1+\frac{\pi q}{4}\right)\left\{1-\left(\frac{1-\frac{\pi q}{4}}{1+\frac{\pi q}{4}}\right)^{n}\right\} \\
& n=\frac{\ln \left(1-A \frac{\pi q}{4} \frac{1}{1+\frac{\pi q}{4}}\right)}{\ln \left(\frac{1-\frac{\pi q}{4}}{1+\frac{\pi q}{4}}\right)}
\end{aligned}
$$

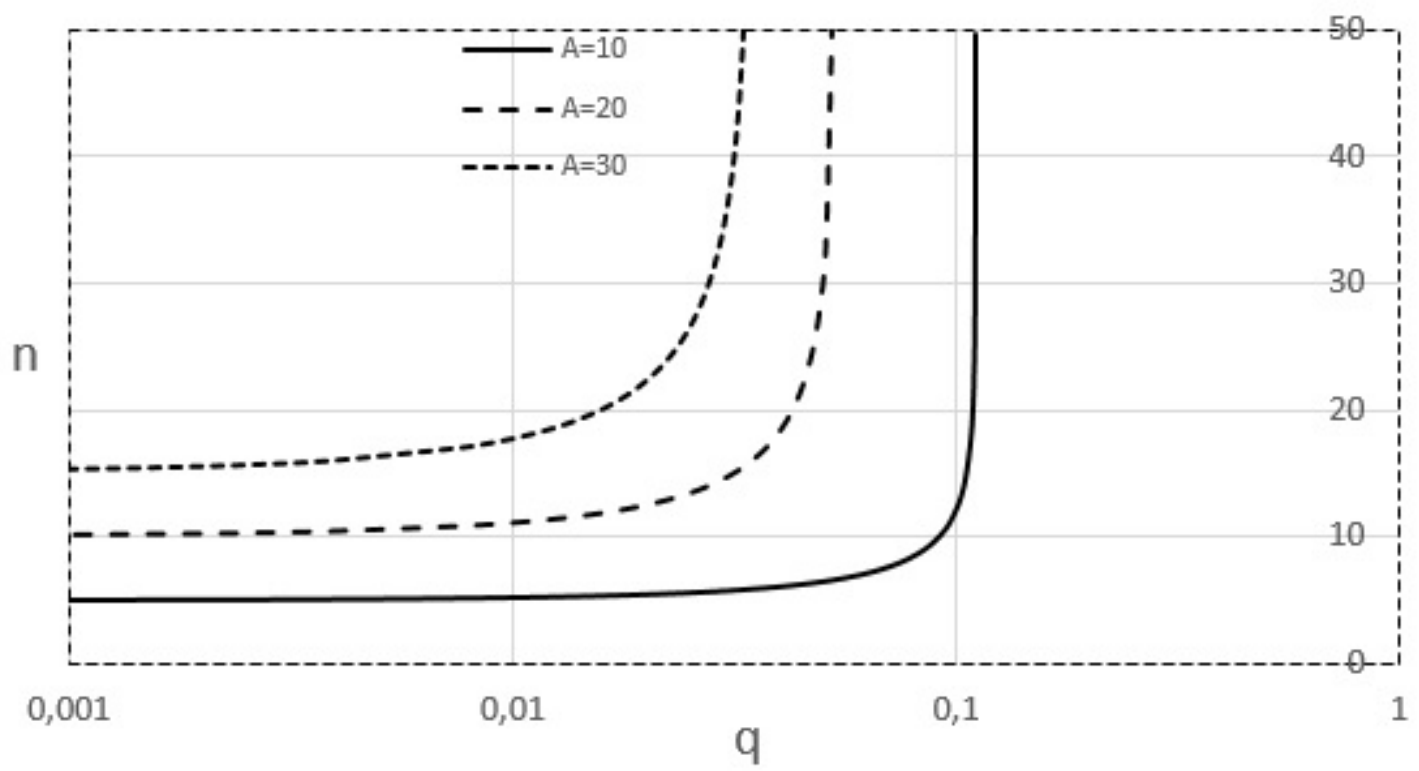

Figure 5. Courbes d'iso-amplitude maximale A en fonction des nombres $n$ et $q$.

La figure 5 présente les courbes d'iso-amplitude maximale en fonction des nombres $n$ (caractéristique de la durée de la tempête) et $q$ (caractéristique de l'élancement du bassin). On remarque que, pour les bassins élancés ( $q$ petit), la durée des tempêtes peut être un facteur limitant. En revanche, pour les bassins peu élancés ( $q$ grand), l'amplitude maximale est indépendante de la durée des tempêtes.

Nous prenons désormais pour référence temporelle $T_{l}=\frac{l}{c}$ la période associée à la largeur de la passe d'entrée.

$$
T=2 n \frac{L}{c}=2 \frac{n}{q} \frac{l}{c}=2 \frac{n}{q} T_{l}
$$

Introduisant le rapport $p=\frac{T}{T_{l}}$, 


\section{XVI èmes Journées Nationales Génie Côtier - Génie Civil \\ Le Havre 2020}

$$
p=\frac{T}{T_{l}}=2 \frac{n}{q}
$$

l'équation (30) se réécrit :

$$
A \approx \frac{4}{\pi q}\left(1+\frac{\pi q}{4}\right)\left\{1-\left(\frac{1-\frac{\pi q}{4}}{1+\frac{\pi q}{4}}\right)^{\frac{p q}{2}}\right\}
$$

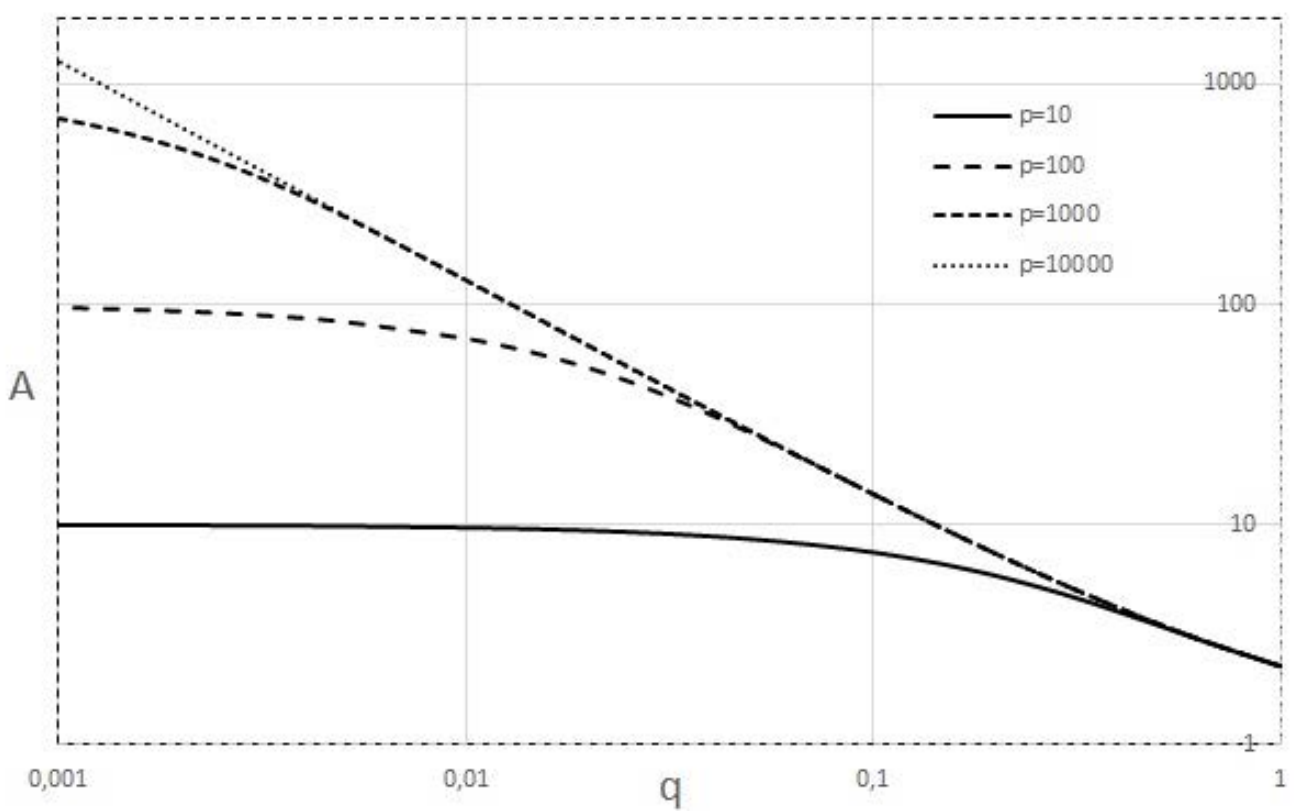

Figure 6. Amplitude maximale A en fonction du nombre q pour une durée de tempête p.

La figure 6 présente différemment les résultats. Elle montre que si $p$ est suffisamment grand, une relation lie l'amplitude maximale à l'élancement du résonateur. En revanche, si $p$ n'est pas suffisamment grand, l'amplitude maximale est limitée en particulier pour les résonateurs élancés. L'amplitude $A$ est bornée par la valeur $p$. L'expression suivante semble une approximation acceptable de (34).

$$
A \approx \min \left\{p, \frac{4}{\pi q}\left(1+\frac{\pi q}{4}\right)\right\}
$$

\section{Conclusions}

Le travail présenté est la suite de travaux visant à trouver des formules analytiques pour la réponse de bassins schématiques à des forçages océano-météorologiques et pour la génération de seiches. Ceci permet d'obtenir une réponse très rapide en amont et en complément de l'utilisation de modèles numériques plus sophistiqués et plus lourds à mettre en œuvre, ce qui est tout à fait pertinent dans le cas d'études sur les conséquences 


\section{Thème 4 - Ouvrages portuaires, offshore et de plaisance}

du changement climatique sur les côtes et sur les installations portuaires. Il faut noter que les modèles numériques rencontrent des difficultés à représenter fidèlement la réponse du résonateur du fait d'une diffusion numérique qui génère un amortissement artificiel de la résonance.

Le travail est ici centré sur la réponse temporelle afin de fournir une méthodologie de calcul pour des forçages instationnaires. L'application des résultats pour un forçage d'une durée fixe montre que la durée de la tempête peut limiter l'amplification de la seiche pour les bassins élancés. Ces travaux pourront être prolongés d'une part à travers la caractérisation des principaux ports français en termes d'amplification maximale et d'autre part à travers l'analyse de leur réponse à des forçages instationnaires.

\section{Références bibliographiques}

PONS F., SERGENT P., LAMBERT A. (2008). Calcul des seiches à partir du logiciel Refonde. $\mathrm{X}^{\mathrm{ème}}$ Journées Nationales Génie Côtier - Génie Civil, Sophia - Antipolis, pp 785-794. https://doi.org/10.5150/jngcgc.2008.074-P

RABINOVICH A.B. (2009). Seiches and harbor oscillations. Handbook of Coastal and Ocean Engineering, World Scientific Publ., Singapore, pp 193-236. https://doi.org/10.1142/9789812819307_0009

SERGENT P., TANGUY J.M., SMAOUI H. (2019). Calcul analytique et numérique des seiches et des oscillations portuaires pour des bassins de forme rectangulaire et de profondeur constante avec des digues semi-infinies parfaitement réfléchissantes. La Houille Blanche, 3-4, pp 117-129. https://doi.org/10.1051/lhb/2019049 